\title{
Effect of Multicomponent Exercise Program on Selected Gait and Balance Parameters in Young Obese Females
}

\author{
Rucha Pravin Kulkarni ${ }^{1}$, Sandeep Babasaheb Shinde ${ }^{2}$ \\ ${ }^{1}$ Department of Musculoskeletal Sciences, Krishna Institute of Medical Sciences (Deemed to \\ be University), Karad, Maharashtra, India. ${ }^{2}$ Department of Musculoskeletal Sciences, \\ Krishna Institute of Medical Sciences (Deemed to be University), Karad, Maharashtra, India.
}

\section{ABSTRACT}

\section{BACKGROUND}

Obesity is a major global health problem. It is now commonly seen in young population due to their lifestyle and unhealthy eating habits. Obesity is now more common in younger females. Due to hormonal changes, body may not adapt, which may lead to life threatening and irreversible conditions. Obesity affects many physical factors including gait and balance. We wanted to study the correlation between obesity and gait-balance parameters, the effect of multi component exercise program on selected gait and balance parameters in young obese females. We also wanted to study its effect on dynamic balance, and compare it with conventional exercise program.

\section{METHODS}

In this comparative study two groups were created with 20 participants with obesity grade 1 BMI in each. Group 2 underwent 26 weeks of multi component exercise. Group 1 underwent conventional exercises. Data was assessed and analysed. Outcome measures were body mass index, base of support, stride length, step length, cadence, and functional reach test.

\section{RESULTS}

Body mass index for group 1 after their conventional exercise decreased in $47 \%$ while in group 2 with multi component exercise protocol BMI reduced in 53\% population. Similarly base of support for group 1 was $42 \%$ and group 2 was $58 \%$; stride length in group 1 was $42 \%$ and group 2 was 58\%; step length for group 1 was $42 \%$ and group 2 was $58 \%$; cadence for group 1 was $42 \%$ and group 2 was $58 \%$; functional reach test was $42 \%$ and $58 \%$ for group 1 and group 2 respectively.

\section{CONCLUSIONS}

A significant improvement was found in selected gait and balance parameters in young obese women with the help of multi component exercise program.

\section{KEY WORDS}

Obesity, Gait, Balance, Body Mass Index, Base of Support, Stride Length, Step Length, Cadence, Functional Reach Test
Corresponding Author: Dr. Sandeep Babasaheb Shinde, Associate Professor, Faculty of Physiotherapy, Krishna Institute of Medical Sciences, Deemed to be University, Karad, Maharashtra, India. E-mail: drsandeepshinde24@gmail.com

DOI: $10.14260 / \mathrm{jemds} / 2020 / 382$

Financial or Other Competing Interests: Dr. Shinde reports grants from Krishna Institute of Medical Sciences, Deemed to be University, Karad, outside the submitted work. Dr. Kulkarni reports grants from Krishna Institute of Medical Sciences, Deemed to be University, Karad, outside the submitted work.

How to Cite This Article: Kulkarni RP, Shinde SB. Effect of multicomponent exercise program on selected gait and balance parameters in young obese females. J. Evolution Med. Dent. Sci. 2020;9(23):1739-1742, DOI: $10.14260 /$ jemds $/ 2020 / 382$

Submission 04-02-2020,

Peer Review 14-05-2020,

Acceptance 20-05-2020,

Published 08-06-2020.

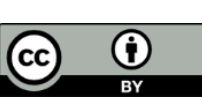




\section{BACKGROUND}

Obesity is a major global health problem. It is now commonly seen in young population due to their lifestyle and unhealthy eating habits. Obesity is now more common in younger females. Due to hormonal changes, body may not adapt, which may lead to life threatening and irreversible conditions. Some of the major problems are gait and balance disturbances. Excessive body weight induces biomechanical burden on the lower extremity affecting the pattern of walking, the speed of walking as compared to the non-obese individuals. ${ }^{[1,2]}$ In these ways the mechanism of obesity is linked with gait. In the similar manner the second most common altered feature is balance. As gait and balance go hand in hand. It is commonly observed that balance impairments are seen due to weak core muscles, so it is very important to train the core muscle with different core muscle strengthening programs also most of the injuries are caused due to fall. It is very difficult for a obese person to maintain their balance during time of hustle which may lead to fall.[3] It may also lead to many injuries in young age. Also due to improper nutrition and junk food habits most of them suffer with low bone mineral density causing frequent fracture due to balance impairments. There are many studies carried on like biomechanical effects of obesity on balance,[4] effects of excessive body weight on postural control,[5] relationship between obesity and injuries among US adult [6], But no study has been carried as an effect to reduce obesity by multi component exercise program in which its effect can be seen and also, we can get rid of this worldwide problem with minimum of risk factors and maximum output by effect of exercises. Obesity is mostly faced by young population. It is necessary to get rid of irreversible diseases such as heart diseases, diabetes, stroke.[7] This study is about the multi component exercise program designed as carried to reduce the prevalence of obesity in young obese females..

According to researches it is found that obese women have a greater net metabolic rate. ${ }^{[8]}$ It is important to get rid of this problem in younger age as if it is carried further it is very difficult for the body to adopt changes and specially in females due to hormonal changes it is very difficult to reduce BMI after crossing their younger age. [9] Exercise is a major game changer in reducing the body mass index. Also, the dietary factors are responsible to increase the calorie count. Maximum weight loss is can be done with proper diet. Also, as a result of biomechanical adaptations to obesity many of the gait and balance parameters are impaired. It is important and challenging from the physiotherapist perspective as they can design multidimensional protocol and can let overcome this problem. Multi-dimensional protocols can be designed for the weight loss program. Diet plays an important role in the weight loss program in this study we have made an effort to keep minimum gap of 7 hours between two meals with two meals per day and a window of 16 hours in between the last meal of the day and first meal of next day. With minimum 2.5 litres of water per day which acts as catalyst in burning the fat as physiological fasting starts the insulin secretion causing burning of fat. Also, similar study was carried on biomechanical effects of obesity on balance. The objective of this review was to analyse the current literature related to effects of obesity on balance. These effects were observed during conditions of static balance, perturbed balance and dynamic balance during gait ${ }^{1}$. In this study we have made to an effort to focus mainly upon the weight loss program which can be performed at home without any gym equipment's and machinery used. So, this study is an effort to find the effect of multi component exercise program in young obese females in selected gait and balance parameters.

\section{METHODS}

An experimental study was carried over a period of 26 weeks. Inclusion criteria were Age group between 18- 25 years, Grade, BMI 30-34.9, exclusion criteria were recent fractures, recent soft tissue injury 3, cardiovascular or respiratory issues. Sampling size included total 40 subjects. Previous studies by Hannah C, Celia M, Darla R, Rebecca J. Biomechanical effects of obesity on balance shown mean \pm standard deviation for BMI for Experimental group is $21 \pm 4.2$ and for control group is $18 \pm 1.6$. Minimum subjects required with $95 \%$ confidence and $80 \%$ power is $n=18$, since follow up study, $n=45+2($ since $10 \%$ attrition rate $)=20$. Hence minimum 20 subjects in either group should be studied.

The subjects were randomly allocated into the two groups of 20 subjects each. Materials used were yoga mat, skipping rope, dumbbells, stopwatch, weighing machine, inch tape, notebook, pen, paper, water bottle, ORS powder. Study was carried in musculoskeletal Outpatient department of Krishna College of physiotherapy.

Ethical approval was taken from the institutional ethical committee of Krishna institute of medical sciences deemed to be university Karad. Consent was taken and study was explained and assessed to the participants personally. With 45 min of session 5 days per week was carried over a period of 26 weeks. Major outcome measures were selected gait and balance parameters. Functional reach test for balance and stride length, step length, cadence and base of support were taken for gait. ${ }^{1}$

1. Group 1 was instructed to follow their routine conventional exercise program. (for example, walking, jogging, etc.)

2. Group 2 the exercise protocol was designed. Which started with warm up session of 10-15 min. which included rolls (head, neck, arms, shoulder, wrist, ankle) also trunk twisting and hip marching. After that skipping (500 skips/ session), slow jumps, high jumps, jumping jacks, lunges, cross lunges, squats, sit ups and planks were included (progression was done in repetition of each exercise). Also, the session concluded with cool down period or shavasana. The participants were instructed to avoid greasy and sweet products and to drink min of 2.5 litres of water daily.

After 26 weeks the participants were assessed personally and compared with the conventional exercise group. Functional reach test for balance and for gait parameters used were stride length, step length, cadence, base of support with BMI. Readings were noted and comparative study was statistically performed. With exercises diet plays a major role in obesity. $70 \%$ of the weight reduction depends on eating habits. For group 1 diet included was 3 meal diet and including breakfast, lunch, and dinner. For group 1 the diet involved was 2 meal diet which included a gap of 7 hours between two meals. With minimum of 2.5 liters of water per day. 


\section{Statistical Analysis}

1. Within Group Comparisons: Within group comparison was done by applying Paired t test to pre and post training values of same group for all the outcome measures.

2. Between the Two Groups: Between the groups, comparison was done by applying Unpaired t- test to pre and post training values of same group for all the outcome measures.

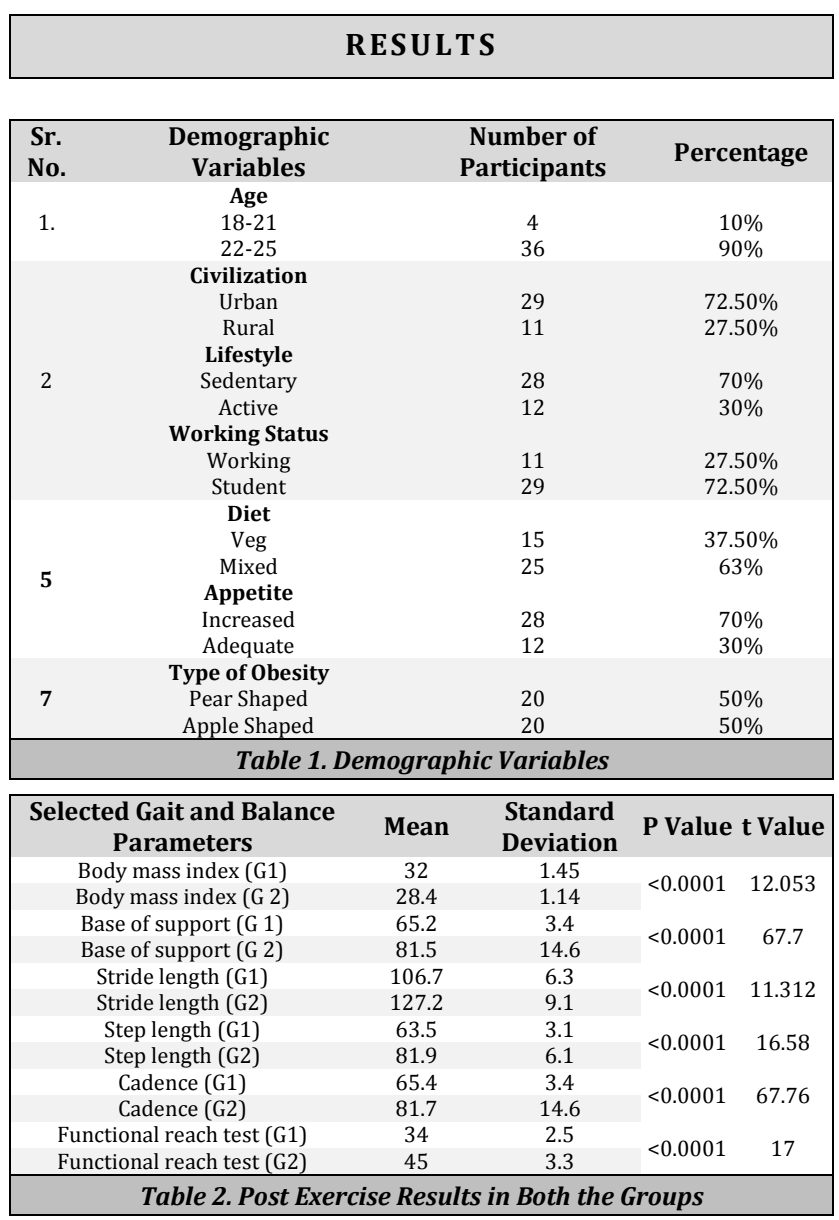

After analysing and comparing the data significant effect was found on young obese females by multi component exercise program with improvement in selected gait and balance parameters. Body mass index, base of support, stride length, step length, cadence, functional reach test were the selected parameters in young obese females with grade 1 obesity. Exercise protocol was given for 26 weeks and before and after Body mass index were assessed the results are as follows. Body mass index for reduced in group 1 (conventional group) by $47 \%$, and reduced in group 2 (multi component exercise protocol) by $53 \%$ respectively. Base of support reduced by $42 \%, 58 \%$ respectively, stride length $42 \%, 58 \%$, step length from $42 \%, 58 \%$, cadence from $42 \%, 58 \%$, and functional reach test from $42 \%, 58 \%$ for group 1 and group 2 respectively. P value was $<0.0001$ which was extremely significant.

\section{DISCUSSION}

The study is about the effect of multi component exercise program on selected gait and balance parameters in young obese females. Obesity is a worldwide problem faced mostly in the younger generation which leads to many irreversible conditions like hypertension, diabetes mellitus, thyroid abnormalities. It is very necessary to reduce this worldwide issue. The objective of this study was to find the effect of multi component exercise program on selected gait and balance variables in young obese females and compare it with the conventional exercise program. Also, major alterations are found in distribution of plantar pressure due to obesity ${ }^{10}$ and speed of walking. Study was carried on 40 young females who were divide in two groups (20 each) with grade 1 obesity according to WHO and over a period of 26 weeks multi component exercise program was given and recorded and compared with the conventional exercise program. Group 1 was instructed to follow their routine conventional exercise program. (for example, walking, jogging, etc.)Group 2 the exercise protocol was designed. Which started with warm up session of 10-15 min. which included rolls (head, neck, arms, shoulder, wrist, ankle) also trunk twisting and hip marching. After that skipping (500 skips / session), slow jumps, high jumps, jumping jacks, lunges, cross lunges, squats, sit ups and planks were included (progression was done in repetition of each exercise). Also, the session concluded with cool down period or shavasana. The participants were instructed to avoid greasy and sweet products and to drink minimum 2.5 litres of water per day. With exercises diet plays a major role in obesity. $70 \%$ of the weight reduction depends on eating habits. For group 1 diet included was 3 meal diet and including breakfast, lunch, and dinner. for group 1 the diet involved was 2 meal diet which included a gap of 7 hours between two meals. Diet plays an important role in the weight loss program in this study we have made an effort to keep minimum gap of 7 hours between two meals with two meals per day with minimum 2.5 litres of water per day which acts as catalyst in burning the fat as physiological fasting starts the insulin secretion causing burning of fat.

Body mass index was reduced in group 1 was $47 \%$ and group 2 was $53 \%$ after the protocol was carried with standard deviation 1.45, 1.14 for G1 and G2 after t value 12.053 and $p$ value $<0.0001$ with extremely significant. Base of support G1 and G2 were $42 \%$ and $58 \%$ respectively with p value of $<0.0001$ and standard deviation of 3.4 and 6.52 respectively and $t$ value of 67.7. stride length for group 1 and group 2 were $42 \%$ and $58 \%$ with standard deviation 6.3 and 9.1 and t value of 11.312 respectively and p value $<0.0001$. Step length $42 \%$ and 58\% with standard deviation 3.1 and 6.1 for group 1 and group 2 respectively with t value 16.58 and $\mathrm{p}$ value $<0.0001$. Cadence $42 \%$ and $58 \%$ with standard deviation of 3.4 and 14.6 with $t$ value 6.7 and $p$ value $<0.0001$ for group 1 and group 2 respectively. For balance parameter functional reach test $42 \%$ and $58 \%$ with standard deviation 2.5 and 3.3 , t value 17 and $p$ value $<0.0001$ for group 1 and group 2 respectively statistical analysis was done to compare the result of both group A and group B Unpaired t test was done for all the components and $p$ value was $<0.0001$ which was significant.. And this shows that there was effect of multi-component exercise program on young obese females. And it can be clinically effective on major obese population with almost no side effects. And physiotherapists can have an upper hand and can design various protocols accordingly. Also, community-based protocols or home protocols can be given also overcome this condition. One example is Therapeutic exercise program is 
effective in improving functional status, grip strength and perceived health in adults with early Rheumatoid arthritis.[11]

To summaries this a study was carried to find the effectiveness of multi component exercise program in young obese females with selected gait and balance parameters. Forty Young females with grade 1 obesity were included and divided into two groups of twenty each. Group one followed their conventional regular exercises and group two multicomponent exercise program was designed and was followed for a period of 26 weeks also two meal diet was given to group two with 7 hours of gap in between two meals and a window period of 16 hours between last meal of the day and first meal of next day. As physiologically insulin secretion and fasting causes fat burning. With all these balance and gait parameters were recorded and analysed and extremely significant results were found with $p$ value $<0.0001$. Physiotherapist play an important role and can design various protocols to get rid of this problem.

\section{CONCLUSIONS}

The study was carried to find the effect of multi component exercise program on young obese females on selected gait and balance parameters. The data was assessed, and a significant decline was found in BMI as well as in gait and balance parameters with $p$ value of $<0.0001$ which was statistically significant.

\section{REFERENCES}

[1] Browning RC, Baker EA, Herron JA, et al. Effects of obesity and sex on the energetic cost and preferred speed of walking. J Appl Physiol 2006;100(2):390-8.

[2] Browning RC, Kram R. Effects of obesity on the biomechanics of walking e different speeds. Med Sci Sport Exer 2007;39(9):1632-41.

[3] Fjeldstad C, Fjeldstad AS, Acree LS, et al. The influence of obesity on falls and quality of life. Dynam Med 2008;7:4.

[4] Hannah C, Celia M, Darla R, et al. Biomechanical effects of obesity on balance. International Journal of Exercise Science 2012;5(4):301-20.

[5] Blaszczyk JW, Cieslinska-Swider J, Elewa M, et al. Effects of excessive body weight on postural control. J Biomech 2009;42(9):1295-300.

[6] Finkelstein EA, Chen H, Prabhu M, et al. The relationship between obesity and injuries among U.S. adults. Am J Health Promot 2007;21(5):460-8.

[7] Himes CL. Obesity, disease and functional limitation in later life. Demography 2000;37(1):73-82.

[8] Browning R, Kram R. Energetic cost and preferred speed of walking in obese vs normal weight women. Obes Res 2005;13(5):891-9.

[9] Greve J, Alomso A, Bordini ACPG, et al. Correlation between body mass index and postural balance. Clinics 2007;62(6):717-20.

[10] Birtane M, Tuna $\mathrm{H}$. The evaluation of plantar pressure distribution in obese and non-obese adults. Clin Biomech 2004;19(10):1055-9.

[11] Shinde SB, Varadharajulu G. Effect of therapeutic exercise programme in adults with early rheumatoid arthritis. Ind J Physiother \& Occup Ther 2017;11(3):76-80. Website: www. ijpot.com. 